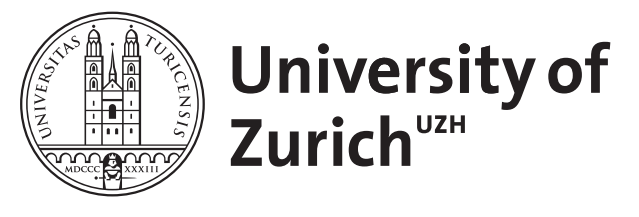

\title{
Der mediale Attraktivitätsbonus
}

\author{
Maurer, Marcus ; Schoen, Harald
}

\begin{abstract}
Zusammenfassung: Der Beitrag untersucht die Berichterstattung sechs regionaler Tageszeitungen über 25 Wahlkreiskandidaten in den letzten sechs Wochen vor der Bundestagswahl 2005 mithilfe einer quantitativen Inhaltsanalyse. In einer vorgeschalteten Studie wurde die physische Attraktivität der 25 Kandidaten ermittelt. Die Analysen zeigen, dass die Zeitungen über attraktive Wahlkreiskandidaten deutlich häufiger und deutlich positiver berichtet haben als über unattraktive. Dies gilt auch dann, wenn man andere für die Berichterstattung relevante Kandidatenmerkmale (Parteizugehörigkeit, Rolle als Amtsinhaber oder Herausforderer usw.) kontrolliert. Die Befunde deuten darauf hin, dass Journalisten denselben Attraktivitätsstereotypen unterliegen wie andere Menschen. Dies hat jedoch vermutlich weitreichende Folgen, weil politische Kandidaten ihre Wahlchancen durch häufige und positive Berichterstattung erheblich verbessern. Die Ursachen und Konsequenzen der Befunde werden diskutiert
\end{abstract}

DOI: https://doi.org/10.1007/s11577-010-0101-6

Posted at the Zurich Open Repository and Archive, University of Zurich

ZORA URL: https://doi.org/10.5167/uzh-156732

Journal Article

Published Version

Originally published at:

Maurer, Marcus; Schoen, Harald (2010). Der mediale Attraktivitätsbonus. Kölner Zeitschrift für Soziologie und Sozialpsychologie, 62(2):277-295.

DOI: https://doi.org/10.1007/s11577-010-0101-6 


\title{
Der mediale Attraktivitätsbonus
}

\section{Wie die physische Attraktivität von Wahlkreiskandidaten die Medienberichterstattung in Wahlkämpfen beeinflusst}

\author{
Marcus Maurer $\cdot$ Harald Schoen
}

Zusammenfassung: Der Beitrag untersucht die Berichterstattung sechs regionaler Tageszeitungen über 25 Wahlkreiskandidaten in den letzten sechs Wochen vor der Bundestagswahl 2005 mithilfe einer quantitativen Inhaltsanalyse. In einer vorgeschalteten Studie wurde die physische Attraktivität der 25 Kandidaten ermittelt. Die Analysen zeigen, dass die Zeitungen über attraktive Wahlkreiskandidaten deutlich häufiger und deutlich positiver berichtet haben als über unattraktive. Dies gilt auch dann, wenn man andere für die Berichterstattung relevante Kandidatenmerkmale (Parteizugehörigkeit, Rolle als Amtsinhaber oder Herausforderer usw.) kontrolliert. Die Befunde deuten darauf hin, dass Journalisten denselben Attraktivitätsstereotypen unterliegen wie andere Menschen. Dies hat jedoch vermutlich weitreichende Folgen, weil politische Kandidaten ihre Wahlchancen durch häufige und positive Berichterstattung erheblich verbessern. Die Ursachen und Konsequenzen der Befunde werden diskutiert.

Schlüsselwörter: Physische Attraktivität · Politische Kandidaten · Medienberichterstattung · Wahlkampf $\cdot$ Inhaltsanalyse

\section{The attractiveness stereotype in the media-How physical attractiveness of political candidates affects newspaper's election coverage}

\begin{abstract}
The study analyses the coverage of six regional newspapers on 25 local candidates during the last six weeks of the 2005 German national election by using quantitative content analysis. In an earlier study the physical attractiveness of the 25 candidates has been examined. The study shows that newspapers covered physically attractive candidates much more often and much more positive than unattractive candidates. This held also true when other relevant candidate characteristics (party membership, role as incumbent or challenger etc.) are controlled. The
\end{abstract}

(C) VS-Verlag 2010

M. Maurer $(\bowtie)$

Institut für Publizistikwissenschaft und Medienforschung, Universität Zürich, Andreasstr. 15, 8050 Zürich, Schweiz

E-Mail: m.maurer@ipmz.uzh.ch

H. Schoen

Lehrstuhl für Politische Soziologie, Otto-Friedrich-Universität Bamberg, Feldkirchenstraße 21,

96045 Bamberg, Deutschland

E-Mail: harald.schoen@uni-bamberg.de 
results show that journalists are victims of the same attractiveness stereotypes as other people. But in the case of journalists there may occur more impotent consequences the amount and tone of media coverage on political candidates may affect the results of election campaigns. Causes and consequences of these findings are discussed.

Keywords: Physical attractiveness $\cdot$ Political candidates $\cdot$ Media coverage $\cdot$ Election campaign . Content analysis

\section{Einleitung}

Physische Attraktivität ist in den Sozialwissenschaften zu einem attraktiven Thema geworden. Forscher unterschiedlicher Disziplinen haben positive Wirkungen physischer Attraktivität auf die Chancen von Menschen in den unterschiedlichsten Lebensbereichen nachgewiesen. So steigert physische Attraktivität erwartungsgemäß die Erfolgsaussichten bei der Partnerwahl (Grammer 2002; Grammer et al. 2003). Ein ansprechendes Äußeres bringt aber auch auf dem Arbeitsmarkt Vorteile und eröffnet bessere Verdienstmöglichkeiten (z. B. Hamermesh u. Biddle 1994; Biddle u. Hamermesh 1998). In der akademischen Welt dürfen physisch attraktive Lehrpersonen damit rechnen, dass ihre Lehrleistung von Studierenden besser bewertet wird (Hamermesh u. Parker 2005; Klein u. Rosar 2006). Auch konnte gezeigt werden, dass attraktive Menschen nicht nur seltener kriminell werden, sondern auch vor Gericht mildere Urteile erwarten dürfen (Efran 1974; Mocan u. Tekin 2010). Untersuchungen in verschiedenen Ländern zeigen schließlich, dass attraktive Bewerber um politische Ämter eher für kompetent gehalten werden und überdurchschnittlich gute Erfolgsaussichten haben oder die Wahlchancen ihrer Parteien zum Teil massiv erhöhen (Efran u. Patterson 1974; Budesheim u. dePaula 1994; C. Sigelman et al. 1986; L. Sigelman et al. 1987; Klein u. Ohr 2000; Klein u. Rosar 2005; Todorov et al. 2005; Berggren et al. 2007; King u. Leigh 2007; Rosar et al. 2008; Lutz 2009).

Attraktivitätseffekte mögen auf allen genannten Gebieten unter ethisch-normativen Gesichtspunkten gleichermaßen fragwürdig erscheinen, doch darf dies nicht den Blick auf einen wichtigen Unterschied verstellen. Während die Effekte auf den anderen Feldern auf persönlichen Kontakten mit mehr oder minder attraktiven Personen beruhen dürften, lernen nur wenige Wahlberechtigte Kandidaten für politische Ämter oberhalb der lokalen Ebene persönlich kennen; die meisten nehmen sie vermittelt über Massenmedien wahr. Die massenmediale Darstellung von Politiker hat erheblichen Einfluss darauf, wie Bürger politische Akteure wahrnehmen und bewerten und für wen sie ihre Stimme abgeben (z. B. Schmitt-Beck 2000; Kepplinger u. Maurer 2005; Grabe u. Bucy 2009). Damit stellt sich die Frage, ob die gut belegten Effekte physischer Attraktivität von Kandidaten auf deren Wahlaussichten mit der Medienberichterstattung zusammenhängen. Eine notwendige Bedingung für einen solchen Zusammenhang besteht darin, dass die physische Attraktivität von Amtsanwärter die Berichterstattung über sie beeinflusst. Damit stellt sich die Frage, inwieweit Journalisten bei ihrer Arbeit den gleichen Wahrnehmungsmechanismen unterliegen, die für Menschen in anderen Lebensbereichen nachgewiesen wurden. Obwohl sich diese Frage vor dem Hintergrund des Forschungsstandes geradezu aufdrängt, liegen dazu unseres Wissens bislang noch keine Analysen vor. Daher werden wir in diesem Aufsatz versuchen, einen Beitrag zu leisten, diese Forschungslücke zu schließen. 
Im Folgenden werden wir zunächst die gängige Argumentation zur Begründung von Attraktivitätseffekten auf die Wahlentscheidung aus der Literatur theoretisch rekonstruieren. Diese Argumentation ergänzen wir um potenzielle Einflüsse der Massenmedien. $\mathrm{Zu}$ diesen entwickeln wir Hypothesen, die wir anschließend empirisch prüfen werden. Abschließend werden die zentralen Ergebnisse der Analyse zusammengefasst und diskutiert.

\section{Theoretische Überlegungen und Forschungsstand}

Arbeiten zur Wirkung der physischen Attraktivität von Kandidaten auf deren Wahlchancen oder die Erfolgsaussichten von deren Parteien greifen in der Regel auf denselben Satz von Beobachtungen und Argumenten zurück, die sie zu einer Argumentationskette zusammenfügen, um Wirkungen physischer Attraktivität zu erklären. Diese Argumentation wollen wir zunächst theoretisch rekonstruieren, ehe wir sie um Medieneffekte ergänzen werden.

Von grundlegender Bedeutung ist der so genannte Attraktivitätskonsens. Demnach sind sich Menschen, auch über Kulturgrenzen hinweg, weitgehend einig darüber, welche Personen als attraktiv gelten und welche nicht. Die Schönheit eines Menschen ist deshalb nicht relativ, wie eine gängige Redensart nahelegt, sondern kann als ein feststehendes Merkmal gelten, das sich relativ eindeutig und objektiv bestimmen lässt (z. B. Gründl 2004, 2007).

Für das Zusammenleben und die Erfolgsaussichten von Menschen ist die physische Attraktivität deshalb wichtig, weil schöne Menschen in verschiedenen Hinsichten Vorteile genießen. Sie ziehen erstens die Aufmerksamkeit anderer Menschen auf sich, erhalten also einen Aufmerksamkeitsbonus (attractiveness attention boost) (z. B. Maner et al. 2003, 2007). Ihnen werden zweitens positiv bewertete Eigenschaften wie Ehrlichkeit, Fleiß, Intelligenz und Kreativität zugeschrieben (attractiveness stereotype). Drittens werden attraktive Menschen in der sozialen Interaktion besser behandelt (attractiveness treatment advantage). Viertens schließlich können sie eher als andere Menschen damit rechnen, dass ihre Fehlleistungen entschuldigt werden (attractiveness glamour effect). In der Kumulation führen diese Einzeleffekte zu einem erheblichen Vorteil, der mit physischer Attraktivität einhergeht. ${ }^{1}$

Der Wettbewerbsvorteil erstreckt sich, wie eingangs verdeutlicht, auch auf die politische Arena, wo attraktive Kandidaten bessere Wahlchancen haben. Um diese Attraktivitätseffekte auf die Wahlchancen zu erklären, werden die skizzierten Argumente auf

1 Es wurde allerdings auch auf einen gegenteiligen Effekt hingewiesen, nach dem mit zunehmender physischer Attraktivität verstärkt geschlechtsspezifische Stereotypisierungen aktiviert werden, so dass sehr attraktive Vertreter des einen Geschlechts in mit dem anderen Geschlecht assoziierten Handlungsfeldern eher mit einem Attraktivitätsmalus als mit einem Attraktivitätsbonus rechnen müssen (Heilman u. Saruwatari 1979; Rennenkampff 2004). Daher könnte man beispielsweise in der nach wie vor eher männlich konnotierten politischen Sphäre für Frauen mit einem negativen Attraktivitätseffekt rechnen. Allerdings ist die empirische Evidenz dazu nicht eindeutig (z. B. Heilman u. Stopeck 1985; Hamermesh u. Parker 2005; Klein u. Rosar 2006; Riniolo et al. 2006; Rosar u. Klein 2009). 
die Wahrnehmung und Bewertung von Kandidaten seitens der Wahlberechtigten angewandt (z. B. Rosar et al. 2008). So wird angenommen, dass attraktive Bewerber eher wahrgenommen und folglich bei der Entscheidung über die Stimmabgabe mit höherer Wahrscheinlichkeit präsent sind. Zweitens wird argumentiert, Wahlberechtigte würden attraktiven Kandidaten auch andere positive Eigenschaften zuschreiben. Zudem sähen sie bei schönen Bewerbern relativ leicht über Fehler und von ihrer eigenen Position abweichende Standpunkte zu politischen Sachfragen hinweg. Und schließlich könnten attraktive Kandidaten damit rechnen, dass unentschlossene Personen sie bei der Stimmabgabe bevorzugen. Im Einklang damit zeigen empirische Untersuchungen zu Bundes- und Landtagswahlen, dass politische Parteien ihr Erststimmen-Wahlergebnis um mehrere Prozentpunkte verbessern, wenn sie statt besonders unattraktiver, ausgesprochen attraktive Wahlkreiskandidaten nominieren (Klein u. Rosar 2005; Rosar et al. 2008). Die Stärke dieses Effekts hängt zudem dergestalt von der Attraktivität der Mitbewerber ab, dass attraktive Kandidaten in einem Feld wenig ansehnlicher Konkurrenten mit einem besonders großen Stimmenbonus rechnen können (Rosar et al. 2008).

Die beträchtlichen Effekte physischer Attraktivität auf die Wahlchancen sind bemerkenswert und werden unter Rückgriff auf unterschiedliche Argumentationsfiguren auf den ersten Blick zureichend erklärt. ${ }^{2}$ Bei genauerem Hinsehen wird jedoch deutlich, dass die Literatur zu Attraktivitätseffekten auf Wahlverhalten eine wichtige Erkenntnis der Forschung zur politischen Kommunikation übersieht: Während Urteile über Stellenbewerber, Angeklagte oder akademisches Lehrpersonal anhand direkter Erfahrungen getroffen werden, nehmen Bürger Kandidaten für politische Ämter vor allem über die Medienberichterstattung vermittelt wahr. Während die Urteilsbildung über Spitzenpolitiker vor allem auf der Berichterstattung der Fernsehnachrichten basiert (Kepplinger u. Maurer 2005, S. 63; Schulz 2007, S. 20), informieren sich Bürger über Wahlkreisbewerber vermutlich vor allem aus regionalen Tageszeitungen, weil Wahlkampfveranstaltungen und Infostände nur von wenigen Wahlberechtigten besucht werden und andere Medien nicht in nennenswerter Weise über Wahlkreisbewerber berichten (Staab 1986).

Weil regionale Tageszeitungen die für Stimmberechtigte wichtigste Quelle der Eindrucksbildung sind, kann man annehmen, dass sie die Beziehung zwischen der physischen Attraktivität von Kandidaten und Wahlverhalten beeinflussen. Dabei kann man zwei mögliche Einflussarten unterscheiden: Regionalzeitungen können zum einen reine Multiplikatoren für die beschriebenen Attraktivitätseffekte sein. Dies wäre dann der Fall, wenn die Zeitungen häufig Fotos von Kandidaten abdruckten, die den Lesern einen Eindruck von der Attraktivität der Bewerber vermitteln. Allerdings enthalten Tageszeitungen in der Regel nur wenige Fotos von politischen Kandidaten, auch wenn deren Zahl zuletzt zugenommen hat (Wilke 2004). Wahrscheinlicher ist deshalb, dass die Regionalzeitungen nicht nur Multiplikatoren, sondern Transformatoren für Attraktivitätseffekte sind: Die Medienberichterstattung über Politik ist kein bloßes Abbild der Realität. Sie basiert

2 Die meisten Studien gehen zumindest implizit davon aus, dass die Attraktivitätseffekte vor allem über Wahlplakate vermittelt werden. Allerdings werden Plakate, sofern sie betrachtet werden, in der Regel mit geringer Aufmerksamkeit genutzt (Schulz et al. 2000; Kühnel et al. 2006, S. 13). Dies lässt es relativ unwahrscheinlich erscheinen, dass die Wähler dezidierte Vorstellungen vom Aussehen der Kandidaten in ihrem Wahlkreis haben. 
zwar auf der Ereignislage, ist aber wesentlich von journalistischen Selektionskriterien und Darstellungsweisen geprägt. Die Journalismusforschung hat eine ganze Reihe von Einflussfaktoren auf journalistische Selektionsentscheidungen identifiziert (zusammenfassend z.B. Esser 1998). Neben den rechtlichen und ökonomischen Bedingungen in einer Gesellschaft oder in einem Mediensystem sind dies vor allem die redaktionellen Linien einzelner Medien und die individuellen Berufsmotive, Einstellungen und Vorlieben einzelner Journalisten.

Von den redaktionellen Linien und den Vorlieben von Journalisten hängt erstens ab, wie viel Aufmerksamkeit Bewerbern in der Berichterstattung gewidmet wird. So wird beispielsweise über die Kandidaten und Aktivitäten großer Parteien häufiger berichtet als über die Kandidaten und Aktivitäten kleinerer Parteien (z. B. Jandura 2007; zusammenfassend Maurer u. Reinemann 2006) und über Amtsinhaber häufiger als über Herausforderer (z. B. Donsbach u. Jandura 2005; zusammenfassend Maurer u. Reinemann 2006, S. 120 ff.). Während der so genannte Amtsbonus häufig damit erklärt wird, dass Amtsinhaber aufgrund ihrer Position mehr Anlass zur Berichterstattung liefern, hat die Bevorzugung größerer Parteien in der Regel nichts damit zu tun, dass die großen Parteien aktiver wären. Sie ist vielmehr die Folge eines journalistischen Konsenses darüber, dass die Aktivitäten größerer Parteien berichtenswerter seien. Solche Effekte sind nicht nur auf der Ebene von Spitzenpolitiker zu beobachten - obwohl sie dort am häufigsten untersucht werden. Sie treten vielmehr in ähnlicher Form auch bei der Berichterstattung regionaler Tageszeitungen über Wahlkreiskandidaten auf (Staab 1986).

Zweitens entscheiden redaktionelle Linien und Vorlieben von Journalisten maßgeblich darüber, mit welcher Tendenz über politische Kandidaten berichtet wird. Hierfür spricht beispielsweise, dass sich die Berichterstattung verschiedener Medien über ein und denselben Politiker zum Teil erheblich unterscheidet (z. B. Noelle-Neumann et al. 1999; Kepplinger u. Maurer 2005). Diese Unterschiede lassen sich nicht mit der Ereignislage erklären, weil der Berichterstattung aller Medien dieselbe Ereignislage zugrunde liegt. Verantwortlich hierfür können vielmehr nur unterschiedliche redaktionellen Linien und Sichtweisen einzelner Journalisten sein.

Wenn wir von einem journalistischen Einfluss auf die Menge und Tendenz der Medienberichterstattung über Wahlkreiskandidaten ausgehen und annehmen, dass Journalisten denselben Wahrnehmungsmechanismen unterliegen wie andere Menschen, können wir damit rechnen, dass attraktive Politiker auch in der Medienberichterstattung bevorzugt werden. Der Aufmerksamkeitsbonus würde dann dazu führen, dass attraktive Menschen eine vergleichsweise hohe Chance haben, überhaupt von Berichterstattern wahrgenommen zu werden. Zudem dürften Journalisten sie intensiver beobachten und sich besser an deren Handlungen und Aussagen erinnern. Damit sollten für attraktive Kandidaten die Chancen steigen, überhaupt Gegenstand der Berichterstattung zu werden und vergleichsweise ausführlich und detailliert dargestellt zu werden. Die Attraktivitätsstereotype sollte dazu führen, dass Journalisten attraktiven Kandidaten positive Eigenschaften zuschreiben, und zwar auch in ihrer Berichterstattung. Drittens sollten Journalisten infolge des attractiveness glamour effect bei physisch attraktiven Bewerbern leichter als bei anderen über Fehlleistungen, fragwürdige Sachpositionen und Diskrepanzen zwischen Wort und Tat hinwegsehen. Der vierte Effekt, der attractiveness treatment advantage, schließlich kann die journalistische Arbeit in verschiedenen Hinsichten beeinflussen: Kommt 
es zu persönlichen Kontakten zwischen Journalisten und Kandidaten, könnten attraktive Bewerber unbewusst bevorzugt behandelt werden. Beispielsweise könnte ihnen mehr Raum für eine positive Selbstdarstellung eingeräumt werden, während weniger attraktive Kandidaten mit gleichsam inquisitorischen Fragen konfrontiert werden.

Zusammengenommen können wir daher festhalten: Sofern Journalisten in den Attraktivitätskonsens einstimmen und sich bei ihrem Handeln von der Attraktivität politischer Kandidaten beeinflussen lassen, müssten attraktive Bewerber mit einer überdurchschnittlich vorteilhaften Berichterstattung rechnen können. Sie kommen insbesondere häufiger in den Medien vor und werden positiver bewertet. Die Tatsache, dass Kandidaten, über die besonders häufig und besonders positiv berichtet wird, bessere Wahlchancen haben als Bewerber, über die nur selten oder überwiegend negativ berichtet wird, ist durch viele Untersuchungen belegt (z. B. Fan u. Tims 1989; Kepplinger u. Maurer 2005; Grabe u. Bucy 2009). Verbindet man beide Annahmen, führt dies zu einem erweiterten Modell des Einflusses der Attraktivität von Kandidaten auf die Wahlentscheidung: Neben direkte Effekte, die man auf persönlichen Kontakt oder die aufmerksame Nutzung von Wahlplakaten zurückführen kann, treten indirekte Effekte, die medienvermittelt sind. Im ersten Schritt führt demnach ein attraktives Äußeres von Kandidaten zu einer intensiveren und positiveren Medienberichterstattung. Im zweiten Schritt führt die intensive und positive Berichterstattung zu einer Verbesserung der Wahlchancen. Direkte und indirekte Einflusswege können zur Erklärung von Attraktivitätseffekten auf Wahlverhalten beitragen, unterscheiden sich allerdings nicht zuletzt in demokratietheoretischer Hinsicht. Denn im ersten Fall findet der Transfer von der physischen Attraktivität auf eine grundsätzlich positive Bewertung in den Köpfen der Wahlberechtigten statt, im zweiten Fall hingegen in den Köpfen von Journalisten. Wahlberechtigte werden dann nur noch mit einer allgemein positiven Berichterstattung konfrontiert, deren Ursache sie nicht nachvollziehen können.

Aus unseren bisherigen Ausführungen ist deutlich geworden, dass es sowohl für Einflüsse der physischen Attraktivität von Kandidaten auf Wahlverhalten als auch für Effekte der Medienberichterstattung auf Wahlverhalten empirische Belege gibt. Wirkungen der physischen Attraktivität von Kandidaten auf die Berichterstattung erscheinen theoretisch plausibel, wurden bislang aber noch nicht untersucht. Wir wollen uns deshalb an dieser Stelle der Frage widmen, ob sich die Berichterstattung von regionalen Tageszeitungen über attraktive und unattraktive Politiker unterscheidet. Wir gehen dabei aufgrund der oben angestellten Überlegungen von zwei zentralen Hypothesen aus:

H1: Regionale Tageszeitungen berichten über attraktive Kandidaten häufiger als über unattraktive.

H2: Regionale Tageszeitungen berichten über attraktive Kandidaten positiver als über unattraktive.

Diese Hypothesen sollen im Folgenden exemplarisch anhand einer Inhaltsanalyse der Berichterstattung über 25 Wahlkreiskandidaten bei der Bundestagswahl 2005 geprüft werden. 


\section{Daten und Methoden}

Basis unserer Analysen sind zum einen Attraktivitätswerte für die Wahlkreiskandidaten von CDU/CSU, SPD, FDP, Grünen und Linkspartei im Bundestagswahlkampf 2005, die von Markus Klein und Ulrich Rosar im Rahmen ihrer Studie zum Einfluss physischer Attraktivität auf den Wahlerfolg erhoben und uns dankenswerter Weise zur Verfügung gestellt wurden. Um die Attraktivität der Kandidaten zu messen, wurden dort 24 studentischen Ratern Porträtfotos der Bewerbern um Bundestagsmandate gezeigt, die im Vorfeld der Wahl von den Webseiten der jeweiligen Parteien kopiert wurden. Die Rater konnten die Fotos am eigenen PC betrachten und wurden vor der Untersuchung nicht darüber informiert, dass es sich bei den dargestellten Personen um Politiker handelt. Die Fotos der Bewerber waren jeweils einzeln und in identischer Größe auf dem Bildschirm zu sehen. Die Rater sollten die Kandidaten im Hinblick auf ihre physische Attraktivität auf einer siebenstufigen bipolaren Skala beurteilen, deren Extrempunkte mit 0 (,unattraktiv“) und 6 (,attraktiv“) bezeichnet waren. Die Urteile der Rater stimmten dem Attraktivitätskonsens entsprechend in hohem Maße überein (Cronbach's Alpha =0,95). Der Mittelwert ihrer Einschätzungen kann folglich als geeignetes Maß für die physische Attraktivität der Kandidaten betrachtet werden.

Da wir zweifellos nicht die Berichterstattung über alle Wahlkreiskandidaten untersuchen können, war es nötig, eine Auswahl zu treffen, die im Wesentlichen von zwei Kriterien geleitet wurde: Zum einen sollten Wahlkreise ausgewählt werden, in denen insgesamt eine gewisse Varianz in der Attraktivität der Kandidaten besteht. Wären die Kandidaten alle gleich oder ähnlich attraktiv, ließen sich Effekte physischer Attraktivität auf die Berichterstattung aus rein statistischen Gründen nicht aufzeigen. Zum anderen sollten Wahlkreise ausgewählt werden, in denen es zwei Tageszeitungen mit unterschiedlichen redaktionellen Linien gibt, um auszuschließen, dass eventuelle Effekte der Attraktivität der Kandidaten tatsächlich auf die Attraktivität und nicht auf die redaktionelle Linie des jeweiligen Blattes zurückzuführen sind. Ausgewählt wurden schließlich fünf Wahlkreise, auf die beide Kriterien zutreffen. Es handelt sich um die Wahlkreise 183 und 184 (Frankfurt I und II), die Wahlkreise 259 und 260 (Stuttgart I und II) sowie den Wahlkreis 207 (Mainz).

Die Attraktivitätswerte der 25 Kandidaten in diesen Wahlkreisen (Mittelwert aller Einschätzungen) variierten zwischen 1,20 und 4,58. Die Kandidaten von Grünen $(2,87)$, CDU $(2,84)$, FDP $(2,79)$ und SPD $(2,63)$ waren im Schnitt etwas attraktiver als die Kandidaten der Linkspartei $(2,25) .{ }^{3}$ Weibliche Kandidaten $(3,26)$ wurden als etwas attraktiver eingeschätzt als männliche $(2,45)$. Allerdings kann man nicht zwingend davon ausgehen, dass die absoluten Attraktivitätswerte für die journalistischen Entscheidungen relevant sind. Vielmehr könnte sich Schönheit als relativ erweisen (z. B. Rosar et al. 2008), weshalb Journalisten bei ihrer Berichterstattung auch davon beeinflusst werden könnten, wie attraktiv ein Bewerber im Vergleich zu seinem lokalen Konkurrenten ist. Um zu prüfen, ob dies der Fall ist, verwenden wir zusätzlich wahlkreisweise zentrierte Attraktivitätswerte. Dazu wurde von jedem Attraktivitätswert das arithmetische Mittel im Wahlkreis

3 Da keine Zufallsstichprobe gezogen wurde, verzichten wir darauf, inferenzstatistische Maße anzugeben. 
abgezogen. Um die Interpretation zu erleichtern, wurden die Werte abschließend auf das Intervall zwischen 0 und 1 reskaliert (arithmetisches Mittel 0,48). ${ }^{4}$

Die Berichterstattung über die Wahlkreiskandidaten wurde in den Lokalteilen von jeweils zwei Tageszeitungen pro Wahlkreis untersucht. In Frankfurt waren dies die Frankfurter Allgemeine Zeitung (Rhein Main Zeitung) und die Frankfurter Rundschau, in Stuttgart die Stuttgarter Zeitung und die Stuttgarter Nachrichten, in Mainz die Mainzer Allgemeine Zeitung und die Mainzer Rheinzeitung. Berichte über bundespolitisch aktive Wahlkreiskandidaten in der überregionalen Berichterstattung wurden nicht erfasst. Der Untersuchungszeitraum umfasste die letzten sechs Wochen vor der Wahl (8. August bis 17. September 2005). Erhoben wurde unter anderem, wie häufig und prominent die Kandidaten in den Zeitungen erwähnt wurden, und wie und anhand welcher Dimensionen sie bewertet wurden. Dazu wurde für jeden Beitrag, in dem ein Kandidat erwähnt wurde, die Tendenz der Darstellung auf einer fünfstufigen Skala erfasst, die von 1 (,eindeutig positiv“) bis 5 (,eindeutig negativ“) reichte. Codiert werden sollte der Gesamteindruck, den ein Beitrag von einem Bewerber hinterlässt. Die Codierung wurde von zwei geschulten Codiererinnen im Herbst 2008 durchgeführt. ${ }^{5}$ Um die Interpretation zu erleichtern, wurde für die Analyse die Bewertung der Kandidaten auf den Wertebereich von 0 (,eindeutig negativ“) bis 1 (,eindeutig positiv“) reskaliert.

\section{Befunde}

In allen sechs Tageszeitungen zusammen genommen erschienen 547 Berichte, in denen mindestens einer der 25 Kandidaten erwähnt wurde. Während über manche Bewerber ausgesprochen häufig berichtet wurde (Maximum: 79 Beiträge), ignorierten die Zeitungen andere weitgehend (Minimum: 5 Beiträge). Zugleich berichteten die Blätter über einige Kandidaten ausgesprochen positiv (bester Wert: 0,89 ) und über andere ausgesprochen negativ (schlechtester Wert: 0,25). ${ }^{6}$

Bislang durchgeführte Untersuchungen zur Berichterstattung über politische Kandidaten legen nahe, dass diese Unterschiede vor allem durch die Parteizugehörigkeit eines Bewerbers und seine Rolle als Amtsinhaber oder Herausforderer erklärbar sind. Unsere Daten stehen im Einklang mit diesen Befunden: Wie häufig über einen Bewerber berichtet wurde, hing ganz erheblich davon ab, welcher Partei er angehört. Über die Kandidaten von SPD und CDU erschienen in den Tageszeitungen durchschnittlich etwa 50 Beiträge, über die der Grünen etwa 40, über die der FDP etwa 25 und über die der Linkspartei sogar

4 Verwendet man wahlkreisweise standardisierte Attraktivitätsmesswerte, resultieren substanziell identische Ergebnisse. Die entsprechenden Resultate sind bei den Autoren erhältlich.

5 Die Intercoderreliablität (einfache paarweise Übereinstimmung) betrug 1,00 für das Vorkommen der Kandidaten und 0,76 für die Tendenz der Kandidatendarstellung. Wir danken Sarah Kirschmann und Marlene Mauk für ihren wesentlichen Beitrag zu dieser Untersuchung.

6 Dagegen erschienen im Untersuchungszeitraum durchschnittlich nur etwa drei Fotos von jedem Kandidaten. Nur insgesamt fünf Beiträge enthielten verbale Hinweise auf die physische Attraktivität einer der Kandidaten. Dies spricht eindeutig dafür, dass die untersuchten Regionalzeitungen nicht als reine Multiplikatoren von Attraktivitätseffekten betrachtet werden können. 
Tab. 1: Menge und Tendenz der Berichterstattung über die Kandidaten nach Parteizughörigkeit

\begin{tabular}{lccccc}
\hline & CDU & SPD & FDP & Grüne & Linkspartei \\
\hline Menge & 47,0 & 53,2 & 26,6 & 38,8 & 17,7 \\
Tendenz & 0,62 & 0,63 & 0,55 & 0,62 & 0,46 \\
\hline
\end{tabular}

Menge: Durchschnittliche Zahl der Artikel über die Kandidaten

Tendenz: Durchschnittliche Bewertung der Kandidaten $(0=$ eindeutig negativ, $1=$ eindeutig positiv)

deutlich weniger als 20. Die Befunde entsprechen folglich dem bekannten Muster, nach dem über Kandidaten der größeren Parteien häufiger berichtet wird als über die Bewerber kleinerer Parteien. Mit der Tendenz der Berichterstattung hing die Parteizugehörigkeit der Kandidaten dagegen kaum zusammen - auch wenn die Kandidaten von FDP und vor allem der Linkspartei etwas schlechter bewertet wurden als die Kandidaten der drei anderen Parteien (Tab. 1).

Auch die Rolle eines Bewerbers als Amtsinhaber oder Herausforderer korrelierte deutlich mit der Berichterstattung über ihn: Während über Amtsinhaber im Schnitt etwa 60mal berichtet wurde, erschienen über Herausforderer oder neue Kandidaten kaum mehr als halb so viele Beiträge. Zugleich wurde über amtierende Wahlkreisabgeordnete auch etwas positiver berichtet als über Herausforderer. Auch dies bestätigt die Befunde vorangegangener Studien, nach denen Amtsinhaber über einen Bonus in der Berichterstattung verfügen.

Wir wollen uns in der vorliegenden Untersuchung allerdings vor allem mit der Frage beschäftigen, welche Rolle die physische Attraktivität der Kandidaten für die Berichterstattung über sie spielt. Dazu haben wir die Daten über die Attraktivität der Kandidaten und über die Berichterstattung über sie in einem Datensatz fusioniert. Dies ermöglicht es, einfache Korrelationen zwischen den beiden Attraktivitätsmaßen (absolute und relative Attraktivität) und der Menge oder Tendenz der Berichterstattung zu berechnen. Tabelle 2 zeigt zunächst, dass die beiden Attraktivitätsmaße zwar relativ hoch miteinander korrelieren, aber nicht deckungsgleich ausfallen. Zudem korreliert die Menge der Berichterstattung über die Kandidaten hoch mit ihrer Tendenz, d. h., dass über Bewerber, über die häufig berichtet wird, auch eher positiv berichtet wird.

Vor allem zeigt Tab. 2 aber, dass die Attraktivität eines Kandidaten erheblich mit der Berichterstattung korreliert ist. Zwischen der Attraktivität eines Bewerbers und der Anzahl der Zeitungsberichte über ihn besteht ein starker Zusammenhang. Die Assoziation wird etwas schwächer, wenn statt der absoluten Attraktivität die relative Attraktivität im Vergleich zu den anderen Kandidaten im Wahlkreis betrachtet wird. Genau umgekehrt verhält es sich bei der Tendenz der Berichterstattung. Hier wird erkennbar, dass vor allem über die Bewerber positiv berichtet wird, die verglichen mit ihren Konkurrenten im Wahlkreis besonders attraktiv sind. Zwischen der absoluten Attraktivität und der Berichterstattungstendenz besteht dagegen kaum ein Zusammenhang.

In den bivariaten Analysen bestätigen sich unsere Annahmen folglich in bemerkenswerter Weise. Wir haben jedoch bereits deutlich gemacht, dass die Menge und die Tendenz der Berichterstattung auch von anderen Faktoren abhängig sind. Wir wollen deshalb die bivariaten Analysen im letzten Schritt um multivariate ergänzen. Die Frage ist dann, ob die Einflüsse der physischen Attraktivität auf die Art der Berichterstattung erhalten 
Tab. 2: Bivariate Korrelationen zwischen den Attraktivitätsindikatoren und der Berichterstattung

\begin{tabular}{llll}
\hline & Relative & & \\
& Attraktivität & Berichterstattungsmenge & Berichterstattungstendenz \\
\hline Attraktivität & 0,69 & 0,46 & 0,09 \\
Relative Attraktivität & - & 0,35 & 0,30 \\
Berichterstattungsmenge & & - & 0,54 \\
\hline
\end{tabular}

Angaben: Pearsons r. Statistische Signifikanzen sind nicht ausgewiesen, weil keine Zufallsstichprobe vorliegt

bleiben, wenn andere Merkmale der Kandidaten kontrolliert werden. Dabei wollen wir zum einen die bereits als besonders einflussreich erkannten Merkmale Parteizugehörigkeit und Rolle als Amtsinhaber oder Herausforderer einbeziehen. Zum anderen soll es um eine Reihe von Kandidatenmerkmalen gehen, deren Einfluss bislang nicht untersucht wurde, aber durchaus plausibel ist. Wir kontrollieren deshalb auch das Geschlecht und das Alter der Bewerber und haben erfasst, welche Ämter sie neben ihrer Kandidatur noch ausüben. Man kann zum Beispiel annehmen, dass über einen Kandidaten häufiger oder positiver berichtet wird, wenn er Mitglied des Bundestages ist, weil er dadurch über eine überregionale Bedeutung verfügt. Zudem kann man annehmen, dass über die Kandidaten häufiger und positiver berichtet wird, die ein lokales überparteiliches (z. B. Landrat) oder Parteiamt (z. B. Kreisvorsitzende) ausüben, weil dann eine gewisse Nähe zu den lokalen Journalisten der Regionalzeitungen plausibel erscheint. ${ }^{?}$

Um die Frage zu beantworten, ob der Einfluss der physischen Attraktivität auf die Menge und die Tendenz der Berichterstattung auch dann bestehen bleibt, wenn die genannten Merkmale der Kandidaten kontrolliert werden, führen wir zwei Paare multipler OLS-Regressionen durch. Zunächst untersuchen wir die Ursachen der Berichterstattungsmenge über die Kandidaten, im zweiten Teil der Analyse die Determinanten der Berichterstattungstendenz. In beiden Analyseschritten verwenden wir die beiden Attraktivitätsmaße in getrennten Regressionen als Prädiktoren. Das erlaubt es uns festzustellen, inwieweit die absolute und die relative Attraktivität für das journalistische Selektionsverhalten relevant sind. Weil gelegentlich angenommen wird, dass sich die physische Attraktivität von Frauen in männlich dominierten Handlungsfeldern wie der Politik auch eher negativ auswirken könnte (beauty-is-beastly-Effekt: Heilman u. Saruwatari 1979; Rennenkampff 2004), berechnen wir sämtliche Regressionsmodelle in einem zweiten Schritt auch in einer Modellvariante mit einem Interaktionsterm aus Geschlecht und physischer Attraktivität.

Tabelle 3 zeigt die Ergebnisse der Analysen zu den Determinanten der Berichterstattungsmenge. Wie man erwarten kann, geht ein erheblicher Einfluss von der Parteizugehörigkeit der Kandidaten aus: über die Bewerber großer Parteien wird deutlich mehr berichtet als über die Kandidaten kleinerer Parteien. Zudem können amtierende Wahlkreisabgeordnete und Kandidaten, die ein überparteiliches Amt auf lokaler Ebene aus-

7 Wir haben darüber hinaus eine Reihe weiterer Merkmale der Kandidaten (z. B. Zugehörigkeit zur Bundesregierung und akademische Titel) erhoben, die jedoch bei den von uns betrachteten Bewerbern so wenig Varianz aufweisen, dass sie nichts zur Erklärung der Berichterstattung beitragen können. 
Tab. 3: Einflüsse auf die Menge der Berichterstattung über einen Kandidaten (lineare Regression)

\begin{tabular}{lrrrrr}
\hline & \multicolumn{2}{c}{ Attraktivität } & & \multicolumn{2}{c}{ Relative Attraktivität } \\
\cline { 2 - 3 } & $\mathrm{b}$ & beta & & $\mathrm{b}$ & beta \\
\hline Mitglied einer großen Partei & 22,90 & 0,61 & & 23,51 & 0,62 \\
Amtsinhaber & 12,82 & 0,19 & & 17,08 & 0,25 \\
Lokales überparteiliches Amt & 5,95 & 0,6 & & 2,97 & 0,08 \\
Lokales Parteiamt & $-8,51$ & $-0,23$ & & $-12,28$ & $-0,33$ \\
Mitglied des Bundestages & $-4,55$ & $-0,12$ & & $-6,67$ & $-0,18$ \\
Geschlecht (wbl.) & $-8,96$ & $-0,22$ & & $-7,00$ & $-0,17$ \\
Alter & $-0,10$ & $-0,07$ & & 0,12 & 0,08 \\
(Relative) Attraktivität & 10,35 & 0,48 & & 31,55 & 0,42 \\
Konstante & 9,54 & & & 14,40 & \\
$\mathrm{R}^{2}$ & 0,62 & & 0,54 & \\
\hline
\end{tabular}

Angegeben sind unstandardisierte und standardisierte Regressionskoeffizienten. Statistische Signifikanzen sind nicht ausgewiesen, weil keine Zufallsstichprobe vorliegt

Basis: Berichterstattung über 25 Wahlkreiskandidaten in 6 regionalen Tageszeitungen

üben, mit einem Aufmerksamkeitsbonus rechnen. Lokale Parteiämter schlagen hingegen negativ zu Buche. Gleiches gilt für ein Bundestagsmandat, wobei zu berücksichtigen ist, dass Direktmandat und die Zugehörigkeit zu einer großen Partei statistisch kontrolliert sind. Schließlich müssen weibliche Kandidaten mit einem medialen Aufmerksamkeitsmalus rechnen.

Spielt neben diesen Merkmalen die physische Attraktivität der Bewerber eine Rolle? Die Antwort lautet eindeutig ja. Die Ergebnisse in Tab. 3 belegen, dass auch unter Kontrolle aller anderen relevanten Kandidatenmerkmale über attraktive Kandidaten deutlich häufiger berichtet wird als über unattraktive. Dies gilt unabhängig davon, ob die absoluten oder relativen Attraktivitätswerte betrachtet werden. Die Bedeutung der physischen Attraktivität unterstreicht ein Blick auf die Beta-Koeffizienten. Sie zeigen, dass die Attraktivität eines Kandidaten die Berichterstattung über sie fast ebenso gut erklären kann wie seine Parteizugehörigkeit. Alle anderen Prädiktoren folgen erst mit deutlichem Abstand.

Es bleibt zu fragen, ob der Attraktivitätseffekt auch substanziell ins Gewicht fällt. Da die Befunde zu beiden Attraktivitätsmaßen zu sehr ähnlichen Ergebnissen führen, beschränken wir die Betrachtung auf die Befunde zur absoluten Attraktivität. Bedenkt man, dass die Attraktivität auf einer von 0 bis 6 reichenden Skala gemessen wird, bedeutet der Regressionskoeffizient von 10,35, dass ein hoch attraktiver Kandidat im Vergleich zu einem sehr unattraktiven Bewerber mit über 60 zusätzlichen Berichten rechnen kann. Allerdings überschätzt dieser Wert das tatsächliche Wirkungspotenzial der Attraktivität in den betrachteten Wahlkreisen. Denn der attraktivste Kandidat in unserer Analyse erzielte einen Attraktivitätswert von 4,58, der am wenigsten attraktive Bewerber einen Messwert von 1,20. Verwendet man die empirischen Minimal- und Maximalwerte, ergibt sich ein Attraktivitätsbonus in Höhe von rund 35 Berichten. Diese Wirkung ist zwar schwächer als der theoretisch mögliche Bonus, jedoch mehr als beachtlich. Gutes Aussehen bringt im Wettbewerb um mediale Aufmerksamkeit demnach einen ähnlich großen Vorteil wie 
die Kombination aus der Mitgliedschaft in einer großen Partei und der Rolle als Wahlkreisabgeordneter $(22,90 * 1+12,82 * 1=35,72)$. Berücksichtigt man, dass in unserer Stichprobe zwischen 5 und 79 Berichte auf die einzelnen Kandidaten entfielen, wird klar, dass ein attraktives Äußeres eines Bewerbers vom Ende der medialen Aufmerksamkeitsskala ins Mittelfeld hieven oder aus diesem an die Spitze der medialen Aufmerksamkeit bringen kann. ${ }^{8}$

Die nach dem Geschlecht differenzierten Befunde in Tab. A1 im Anhang deuten auf geschlechtsspezifische Unterschiede in der Stärke des attraktivitätsbedingten Aufmerksamkeitsbonus hin. Für Männer resultiert zwar ein beträchtlicher Regressionskoeffizient von 7,94, bei Frauen ist der entsprechende Koeffizient jedoch rund doppelt so groß $(7,94+8,92=16,86)$. Berücksichtigt man die Verteilung der Attraktivitätswerte unter den untersuchten Kandidatinnen und Kandidaten, zeigt sich, dass die attraktivste Kandidatin im Vergleich zu ihrer am wenigsten attraktiven Mitbewerberin mit einem Aufmerksamkeitsbonus von knapp 38 Berichten rechnen kann, während unter den männlichen Bewerbern der entsprechende Effekt mit rund 23 Berichten noch immer beträchtlich, aber doch deutlich kleiner ist. Physische Attraktivität scheint demnach bei Kandidatinnen noch stärker als bei männlichen Bewerbern die journalistische Aufmerksamkeit zu steigern.

Die Befunde zu den Determinanten der Tendenz der Berichterstattung in Tab. 4 zeigen, dass Mitglieder großer Parteien und amtierende Wahlkreisabgeordnete mit einer vergleichsweise positiven Berichterstattung rechnen können. Dagegen gehen lokale Parteiämter mit einem deutlichen Malus in der journalistischen Bewertung einher. Schwächere negative Effekte gehen von einem Bundestagsmandat und dem Lebensalter aus, während lokale überparteiliche Ämter und das Kandidatengeschlecht ohne konsistente Wirkung bleiben. Bis hierher zeigt sich somit, dass die Faktoren, die die mediale Aufmerksamkeit steigern, cum grano salis auch eine positive Bewertung begünstigen.

Doch gilt dies auch für die physische Attraktivität? Die Ergebnisse zur absoluten und relativen Attraktivität geben darauf unterschiedliche Antworten. Betrachtet man die absolute Attraktivität, lässt sich praktisch kein Effekt nachweisen. Anders im Falle der relativen Attraktivität: Gemessen an den Beta-Koeffizienten, gehört sie wiederum zu den erklärungskräftigen Prädiktoren. Diese Schlussfolgerung erhärtet zusätzlich ein Blick auf die unstandardisierten Regressionskoeffizienten. Der entsprechende Koeffizient bedeutet, dass ein (relativ) attraktiver Kandidat im Vergleich zu einem unattraktiven Bewerber einen Bonus von 0,26 und damit von einem Viertel des Wertebereichs der Kandidatenbewertung erhält. Damit fällt der Attraktivitätsvorteil etwas stärker ins Gewicht als der Bonus, den Inhaber eines Direktmandats in der journalistischen Darstellung genießen. Auch im Hinblick auf die Bewertung der Kandidaten erweist sich die physische Attraktivität somit als ein gewichtiger Faktor.

Allerdings gilt dies nur dann, wenn man die relative Attraktivität betrachtet, nicht hingegen, wenn man die absoluten Attraktivitätswerte heranzieht. Dieser Befund deutet darauf hin, dass die Effekte der physischen Attraktivität dadurch zustande kommen, dass Journalisten (unbewusst) Vergleiche zwischen den Bewerbern innerhalb eines Wahlkrei-

8 Dieser Befund gilt für relative Attraktivität praktisch unverändert, während deren Wirkung im Vergleich zum kumulativen Effekt einer Parteimitgliedschaft und eines Direktmandats etwas schwächer ausfällt. 
Tab. 4: Einflüsse auf die Tendenz der Berichterstattung über einen Kandidaten (lineare Regression)

\begin{tabular}{|c|c|c|c|c|}
\hline & \multicolumn{2}{|c|}{ Attraktivität } & \multicolumn{2}{|c|}{ Relative Attraktivität } \\
\hline & $\mathrm{b}$ & beta & $\mathrm{b}$ & beta \\
\hline Mitglied einer großen Partei & 0,07 & 0,20 & 0,10 & 0,26 \\
\hline Amtsinhaber/in & 0,18 & 0,27 & 0,19 & 0,31 \\
\hline Lokales überparteiliches Amt & 0,02 & 0,06 & $-0,01$ & $-0,03$ \\
\hline Lokales Parteiamt & $-0,16$ & $-0,46$ & $-0,18$ & $-0,50$ \\
\hline Mitglied des Bundestages & $-0,02$ & $-0,06$ & $-0,04$ & $-0,10$ \\
\hline Geschlecht (wbl.) & 0,02 & 0,04 & $-0,05$ & $-0,10$ \\
\hline Alter & $-0,005$ & $-0,33$ & $-0,002$ & $-0,19$ \\
\hline (Relative) Attraktivität & 0,001 & 0,004 & 0,26 & 0,33 \\
\hline Konstante & 0,85 & & 0,64 & \\
\hline $\mathrm{R}^{2}$ & 0,28 & & 0,36 & \\
\hline
\end{tabular}

Angegeben sind unstandardisierte und standardisierte Regressionskoeffizienten. Statistische Signifikanzen sind nicht ausgewiesen, weil keine Zufallsstichprobe vorliegt

Basis: Berichterstattung über 25 Wahlkreiskandidaten in 6 regionalen Tageszeitungen

ses anstellen. Anders gewendet: Die Wirkung des Äußeren eines Wahlkreiskandidaten auf die Berichterstattung im Lokaljournalismus hängt auch davon ab, wie attraktiv seine direkten Konkurrenten um das Direktmandat sind.

Wie die Ergebnisse in Tab. A2 im Anhang zeigen, hängt auch der Einfluss der physischen Attraktivität auf die Tendenz der Berichterstattung vom Geschlecht der Bewerber ab. Allerdings unterscheidet sich dieser Effekt gravierend von jenem, den wir in der Analyse des Umfangs der Berichterstattung festgestellt haben. Bei männlichen Bewerbern sorgt physische Attraktivität für eine deutlich wohlmeinendere Berichterstattung. Dieser Bonus beläuft sich auf beinahe ein Drittel des Wertebereichs der Kandidatenbewertung $(b=0,30)$. Bei Kandidatinnen resultiert hingegen ein leicht negativer Effekt von $-0,09$ $(0,30-0,39)$. Während also männliche Bewerber für physische Attraktivität mit einer positiven Berichterstattung belohnt wurden, konnten Frauen keinen Bonus verbuchen, sondern mussten eher einen Malus hinnehmen. In der Tendenz spricht dieser Befund für einen zumindest moderaten beauty is beastly-Effekt.

Unsere empirischen Befunde sprechen zusammengenommen dafür, dass über attraktive Kandidaten, mit geschlechtsspezifischen Nuancierungen, vergleichsweise häufig und positiv berichtet wird. Sofern Wahlberechtigte die Berichterstattung zur Kenntnis nehmen, dürften ihnen attraktive Bewerber eher im Gedächtnis präsent sein. Zugleich dürften sie einen besseren Eindruck von ihnen besitzen als von weniger attraktiven Amtsanwärtern - und zwar auch dann, wenn sie keinen einzigen Bewerber persönlich kennengelernt oder auf Plakaten wahrgenommen haben. Da Bewertungen von Kandidaten als eine wichtige Determinante der Wahlentscheidung gelten (z. B. Schoen u. Weins 2005, S. 234-240), sind die Voraussetzungen für einen medienvermittelten Stimmenbonus für physisch attraktive Kandidaten erfüllt. 


\section{Zusammenfassung und Diskussion}

Ausgangspunkt unserer Überlegungen waren Befunde, nach denen die Attraktivität politischer Kandidaten deren Wahlchancen erheblich erhöht. Dies wird in der Wahlforschung üblicherweise als direkter Effekt betrachtet: Die Wahlberechtigten kennen die Kandidaten beispielsweise von Wahlplakaten, erinnern sich an attraktive Kandidaten besser und erliegen dem allzu menschlichen Schluss, nach dem attraktive Menschen auch andere positive Persönlichkeitseigenschaften besitzen. Weil die meisten Menschen Wahlkreisbewerber aber vor allem aus den Massenmedien kennen, haben wir einen zweiten, indirekten Einflussweg vermutet: Die Attraktivität der Bewerber wirkt auf das Wahlverhalten dadurch, dass sie die Berichterstattung über die Kandidaten beeinflusst, die ihrerseits Wahlberechtigten als eine wesentliche Grundlage für die Meinungsbildung dient. Eine zentrale Rolle in dieser Argumentation spielt die Annahme, dass Journalisten denselben Mechanismen der Urteilsbildung unterliegen wie die Wahlberechtigten: Sie widmen attraktiven Kandidaten mehr Aufmerksamkeit und bewerten sie positiver als unattraktive Bewerber. Diese Urteile schlagen sich in der Berichterstattung nieder, weil Redaktionen und einzelne Journalisten einen relativ großen Einfluss auf die Selektion von Ereignissen und die Tendenz der Berichterstattung besitzen. Wir haben deshalb vermutet, dass über attraktive Wahlkreiskandidaten häufiger und positiver berichtet wird als über unattraktive.

Diese Vermutungen haben sich in unserer Analyse der Berichterstattung über die Wahlkreiskandidaten im Bundestagswahlkampf 2005 in den Lokalteilen sechs regionaler Tageszeitungen bestätigt. Lokaljournalisten berichten über attraktive Kandidaten deutlich häufiger als über unattraktive. Zudem stellen sie die attraktiveren Kandidaten ihres Wahlkreises positiver dar als die unattraktiveren. Diese Befunde bleiben auch dann erhalten, wenn man andere, für die Berichterstattungsmenge oder -tendenz relevante Merkmale der Kandidaten kontrolliert. Darüber hinaus wird deutlich, dass kaum ein anderes Merkmal der Kandidaten die Menge und Tendenz der Berichterstattung so gut erklären kann wie die physische Attraktivität. Dabei spielt die relative Attraktivität im Vergleich zu den Konkurrenten im Wahlkreis eine ähnliche (Berichterstattungsmenge) oder sogar größere (Berichterstattungstendenz) Rolle als die absolute Attraktivität. Dies deutet darauf hin, dass Journalisten unbewusste Vergleiche zwischen den direkten Konkurrenten um die Gunst der Wahlberechtigten anstellen, die sich dann in der Berichterstattung niederschlagen.

Bedenkt man, dass sich Personalmanager, Richter, Studierende und Wahlberechtigte der Anziehungskraft der physischen Attraktivität nicht entziehen können, ist es keineswegs überraschend, dass dies auch für Journalisten gilt. Die häufige und positive Berichterstattung über attraktive Politiker dürfte demnach nicht die Folge bewusster Selektionsentscheidungen sein. Vielmehr scheinen Journalisten schlicht denselben Wahrnehmungen und Wahrnehmungsverzerrungen wie alle Menschen zu unterliegen. Dies ist kein neuer Gedanke. Auch die Nachrichtenfaktoren, also die Kriterien, nach denen Journalisten auswählen, über welche Ereignisse sie berichten, sind als allgemeinmenschliche Wahrnehmungskriterien konzipiert - auch wenn die Wahrnehmungen von Journalisten und Rezipienten bisweilen deutlich voneinander abweichen (zuletzt Fretwurst 2008).

Die Tatsache, dass auch Journalisten verschiedenen Attraktivitätsstereotypen unterliegen, hat jedoch vermutlich weitreichende Konsequenzen: Die Häufigkeit und die Tendenz, 
mit der die Massenmedien über politische Kandidaten berichten, hat einen erheblichen Einfluss auf die Urteile von Wahlberechtigten über Kandidaten und letztlich auch auf ihre Wahlentscheidungen. Unsere Befunde stehen folglich in Einklang mit der Annahme, dass die Tatsache, dass attraktive Kandidaten bessere Wahlchancen haben, nicht nur auf die direkten Wahrnehmungen der Kandidatenattraktivität durch die Wähler, sondern zumindest zum Teil auch darauf zurückzuführen ist, dass attraktive Kandidaten bessere Chancen auf (positive) Medienpräsenz haben. Die beiden konkurrierenden Erklärungsmodelle müssen vor allem deshalb sorgfältig getrennt werden, weil sie sich in der Antwort auf die demokratietheoretisch relevante Frage unterscheiden, an welcher Stelle der Transfer von der Wahrnehmung der physischen Attraktivität zu einer allgemein positiven Bewertung erfolgt: in den Köpfen der Wahlberechtigten oder in den Köpfen der Journalisten. Fände er (vor allem) in den Köpfen der Journalisten statt, wäre dies ein weiterer Beleg für erhebliche Medieneinflüsse in Wahlkämpfen, die weit über die bloße Vermittlung von Ereignissen hinausgehen. Ob und wie stark der journalistische Attraktivitätsbonus die Wirkung physischer Attraktivität auf die Wahlentscheidung tatsächlich bestimmt, sollte deshalb in künftigen Analysen untersucht werden, die die konkurrierenden Erklärungsmodelle simultan betrachten.

Unsere Analyse hat darüber hinaus geschlechtsspezifische Unterschiede in der Ausprägung von Attraktivitätseffekten ergeben. Physische Attraktivität von Bewerbern steigert die Häufigkeit der Berichterstattung, doch fällt dieser Aufmerksamkeitsbonus bei Kandidatinnen besonders groß aus. Im Gegensatz dazu profitieren männliche Bewerber von ihrer physischen Attraktivität deutlich in Form vergleichsweise wohlmeinender Berichterstattung, nicht jedoch Kandidatinnen, die in dieser Hinsicht zumindest mit einem leichten Malus rechnen müssen. Der Befund zur Tendenz der Berichterstattung lässt sich als Hinweis auf einen (tendenziellen) beauty is beastly-Effekt deuten. Demnach aktiviert physische Attraktivität auch geschlechtsspezifische Stereotypisierungen, so dass attraktiven Frauen verstärkt feminine Eigenschaften zugeschrieben werden, was Bewerberinnen im männlich konnotierten politischen Kontext zum Nachteil gereicht und Zweifel an deren Kompetenz nährt. Das Auseinanderfallen von prononciert wahrgenommenen femininen Eigenschaften der Kandidatinnen und den Anforderungen eines vorwiegend männlich konnotierten Handlungskontexts könnte auch dazu beitragen, die geschlechtsspezifischen Unterschiede des attraktivitätsbedingten Aufmerksamkeitsbonus zu erklären. Denn eine solche Diskrepanz fügt sich sehr gut zur journalistischen Tendenz, bevorzugt über überraschende und außergewöhnliche Begebenheiten zu berichten.

Die Aussagekraft unserer Analyse unterliegt allerdings einigen Beschränkungen. Wir haben unsere Untersuchung auf einer relativ schmalen Basis durchgeführt, weil wir die Berichterstattung der unzähligen Regionalzeitungen in Deutschland nicht vollständig untersuchen konnten. Dies hat zu einigen Problemen geführt: Unsere Stichprobe umfasst nur 25 Wahlkreiskandidaten, wir konnten deshalb verschiedene, vermutlich allerdings weniger relevante, Kandidatenmerkmale nicht in unsere Analyse aufnehmen. Zudem konnten wir eine Reihe von Berichterstattungsmerkmalen, die wir erhoben haben, nicht sinnvoll in die Analyse einbeziehen, weil die Fallzahlen zu gering waren. Dabei geht es beispielsweise um die Frage, ob attraktive Kandidaten auch häufiger und positiver im Bild gezeigt werden oder ob sich die Darstellung attraktiver Bewerber auch auf die Darstellung ihrer Parteien auswirkt. Auch könnte man die Berücksichtigung weiterer Kontrollvaria- 
blen fordern. Es ist jedoch zu bedenken, dass dies die dargestellten Attraktivitätseffekte nur dann abschwächte, wenn die Kontrollvariablen sowohl mit der Attraktivität als auch mit den jeweiligen abhängigen Variablen zusammenhingen. So ist z. B. die journalistische Nachrichtenauswahl auch bei der Berichterstattung über Wahlkreiskandidaten von Nachrichtenfaktoren wie Überraschung oder Negativität beeinflusst. Dies würde den Effekt der physischen Attraktivität auf die Berichterstattung aber nur verringern, wenn physisch attraktive Kandidaten systematisch häufiger an überraschenden oder negativen Ereignissen beteiligt wären. Dies erscheint allerdings ausgesprochen unwahrscheinlich. Zudem kann man einwenden, dass die Befunde möglicherweise nicht in allen Wahlkreisen und allen anderen Wahlen genau so ausgefallen wären. Dies sollte in zukünftigen Studien mit größeren Stichproben untersucht werden. Auch wenn man diese Einschränkungen der Aussagekraft in Rechnung stellt, erscheinen unsere Befunde aus mindestens zwei Gründen dennoch bedeutsam: Aus Sicht der Journalismusforschung liefern sie Einblicke in „,irrationale“ Berichterstattungsursachen, die so bislang noch nicht diskutiert wurden. Aus Sicht der Wahlforschung liefern sie einen neuen Baustein für eine Theorie zum Einfluss physischer Attraktivität auf die Wahlentscheidung.

\section{Anhang}

Tab. A1: Einflüsse auf die Menge der Berichterstattung über einen Kandidaten mit Interaktionsterm aus Attraktivität und Geschlecht (lineare Regression)

\begin{tabular}{|c|c|c|c|c|}
\hline & \multicolumn{2}{|c|}{ Attraktivität } & \multicolumn{2}{|c|}{ Relative Attraktivität } \\
\hline & $\mathrm{b}$ & beta & $\mathrm{b}$ & beta \\
\hline Mitglied einer großen Partei & 23,82 & 0,63 & 24,45 & 0,65 \\
\hline Amtsinhaber/in & 6,19 & 0,09 & 14,18 & 0,21 \\
\hline Lokales überparteiliches Amt & 6,27 & 0,17 & 2,21 & 0,06 \\
\hline Lokales Parteiamt & $-8,34$ & $-0,23$ & $-11,81$ & $-0,32$ \\
\hline Mitglied des Bundestages & $-5,33$ & $-0,14$ & $-6,53$ & $-0,18$ \\
\hline Geschlecht (wbl.) & $-35,54$ & $-0,86$ & $-17,64$ & $-0,43$ \\
\hline Alter & $-0,15$ & $-0,10$ & 0,11 & 0,07 \\
\hline (Relative) Attraktivität & 7,94 & 0,37 & 29,64 & 0,39 \\
\hline Geschlecht * (relative) Attraktivität & 8,92 & 0,73 & 18,05 & 0,28 \\
\hline Konstante & 17,97 & & 15,91 & \\
\hline $\mathrm{R}^{2}$ & 0,64 & & 0,55 & \\
\hline
\end{tabular}

Angegeben sind unstandardisierte und standardisierte Regressionskoeffizienten. Statistische Signifikanzen sind nicht ausgewiesen, weil keine Zufallsstichprobe vorliegt

Basis: Berichterstattung über 25 Wahlkreiskandidaten in 6 regionalen Tageszeitungen 
Tab. A2: Einflüsse auf die Tendenz der Berichterstattung über einen Kandidaten mit Interaktionsterm aus Attraktivität und Geschlecht (lineare Regression)

\begin{tabular}{lccccr}
\hline & \multicolumn{2}{c}{ Attraktivität } & & \multicolumn{2}{c}{ Relative Attraktivität } \\
\cline { 2 - 3 } & $\mathrm{b}$ & beta & & b & beta \\
\hline Mitglied einer großen Partei & 0,08 & 0,22 & & 0,08 & 0,23 \\
Amtsinhaber/in & 0,14 & 0,22 & & 0,25 & 0,38 \\
Lokales überparteiliches Amt & 0,02 & 0,06 & & 0,01 & 0,03 \\
Lokales Parteiamt & $-0,16$ & $-0,45$ & & $-0,19$ & $-0,54$ \\
Mitglied des Bundestages & $-0,02$ & $-0,07$ & & $-0,04$ & $-0,12$ \\
Geschlecht (wbl.) & $-0,13$ & $-0,32$ & & 0,18 & 0,46 \\
Alter & $-0,005$ & $-0,35$ & & $-0,002$ & $-0,14$ \\
(Relative) Attraktivität & $-0,01$ & $-0,06$ & & 0,30 & 0,42 \\
Geschlecht * (relative) Attraktivität & 0,05 & 0,41 & & $-0,39$ & $-0,63$ \\
Konstante & 0,89 & & & 0,61 & \\
$\mathrm{R}^{2}$ & 0,28 & & & 0,37 & \\
\hline
\end{tabular}

Angegeben sind unstandardisierte und standardisierte Regressionskoeffizienten. Statistische Signifikanzen sind nicht ausgewiesen, weil keine Zufallsstichprobe vorliegt

Basis: Berichterstattung über 25 Wahlkreiskandidaten in 6 regionalen Tageszeitungen

\section{Literatur}

Berggren, Niclas, Henrik Jordahl, und Panu Poutvaara. 2007. The looks of a winner: Beauty, gender and electoral success. München: CESifo.

Biddle, Jeff E., und Daniel S. Hamermesh. 1998. Beauty, productivity, and discrimination: Lawyers' looks and lucre. Journal of Labor Economics 16:172-201.

Budesheim, Thomas L., und Stephen J. DePaula. 1994. Beauty or the beast? The effects of appearance, personality, and issue information on evaluations of political candidates. Personality and Social Psychology Bulletin 20:339-348.

Donsbach, Wolfgang, und Olaf Jandura. 2005. Rückkehr des Kanzlerbonus. Redepräsenz der Kanzlerkandidaten in den Fernsehnachrichten. In Wählerstimmungen in der Mediendemokratie. Analysen auf Basis des Bundestagswahlkampfs 2002, Hrsg. Elisabeth Noelle-Neumann, Wolfgang Donsbach, und Hans Mathias Kepplinger, 69-90. Freiburg: Alber.

Efran, Michael G. 1974. The effect of physical appearance on the judgment of guilt, interpersonal attraction, and severity of recommended punishment in simulated jury task. Journal of Research in Personality 8:45-54.

Efran, Michael G., und E. W. J. Patterson. 1974. Voters vote beautiful: The effect of physical appearance on a national election. Behavioral Science 6:352-356.

Esser, Frank. 1998. Die Kräfte hinter den Schlagzeilen. Englischer und deutscher Journalismus im Vergleich. Freiburg: Alber.

Fan, David P., und Albert R. Tims. 1989. The impact of news media on public opinion. American presidential election 1987-88. International Journal of Public Opinion Research 1:151-163.

Fretwurst, Benjamin. 2008. Nachrichten im Interesse der Zuschauer. Eine konzeptionelle und empirische Neubestimmung der Nachrichtenwerttheorie. Konstanz: UVK.

Grabe, Maria Elizabeth, und Erik Page Bucy. 2009. Image bite politics. News and the visual framing of elections. New York: Oxford University Press.

Grammer, Karl. 2002. Signale der Liebe. Die biologischen Gesetze der Partnerschaft. Frankfurt a.M.: dtv. 
Grammer, Karl, Bernhard Fink, Anders P. Møller, und Randy Thornhill. 2003. Darwinian aesthetics: Sexual selection and the biology of beauty. Biological Review 78:385-407.

Gründl, Martin. 2004. Was ist Schönheit? In Bodytalk. Der riskante Kult um Körper und Schönheit, Hrsg. Andrea Hauner und Elke Reichart, 9-33. Frankfurt a.M.: dtv.

Gründl, Martin. 2007. Attraktivitätsforschung: Auf der Suche nach der Formel für Schönheit. In Die Macht der Schönheit, Hrsg. Cathrin Gutwald und Raimar Zons, 49-70. München: Fink.

Hamermesh, Daniel S., und Jeff E. Biddle. 1994. Beauty and the labour market. American Economic Review 84:1174-1194.

Hamermesh, Daniel S., und Amy M. Parker. 2005. Beauty in the classroom. Professors' pulchritude and putative pedagogical productivity. Economics of Education Review 24:369-376.

Heilman, Madeline E., und Luis R. Saruwatari. 1979. When beauty is beastly: The effects of appearance and sex on evaluations of job applicants for managerial and nonmanagerial jobs. Organizational Behavior and Human Decision Processes 23:360-372.

Heilman, Madeline E., und Melanie H. Stopeck. 1985. Being attractive, advantage or disadvantage? Organizational Behavior and Human Performance 35:202-215.

Jandura, Olaf. 2007. Kleinparteien in der Mediendemokratie. Wiesbaden: VS-Verlag.

Kepplinger, Hans Mathias, und Marcus Maurer. 2005. Abschied vom rationalen Wähler. Warum Wahlen im Fernsehen entschieden werden. Freiburg: Alber.

King, Amy, und Andrew Leigh. 2007. Beautiful politicians. Working paper. Adelaide: University of South Australia.

Klein, Markus, und Dieter Ohr. 2000. Gerhard oder Helmut: „Unpolitische“ Kandidateneigenschaften und ihr Einfluß auf die Wahlentscheidung bei der Bundestagswahl 1998. Politische Vierteljahresschrift 41:199-224.

Klein, Markus, und Ulrich Rosar. 2005. Physische Attraktivität und Wahlerfolg. Eine empirische Analyse am Beispiel der Wahlkreiskandidaten bei der Bundestagswahl 2002. Politische Vierteljahresschrift 46:263-287.

Klein, Markus, und Ulrich Rosar. 2006. Das Auge hört mit! Der Einfluss der physischen Attraktivität des Lehrpersonals auf die studentische Evaluation von Lehrveranstaltungen - eine empirische Analyse am Beispiel der Wirtschafts- und Sozialwissenschaftlichen Fakultät der Universität zu Köln. Zeitschrift für Soziologie 35:305-316.

Kühnel, Steffen M., Oskar Niedermayer, und Bettina Westle. 2006. Deutsche Bundestagswahlstudie 2005 , Bürger und Parteien in einer veränderten Welt“. Codebuch 2006. Köln.

Lutz, Georg. 2009. The electoral success of beauties and beasts. FORS Working papers 2009-2. Lausanne.

Maner, Jon K., Douglas T. Kenrick, Vaughn D. Becker, Andreas W. Delton, Brian Hofer, Chris J. Wilbur, und Steven L. Neuberg. 2003. Sexually selective cognition: Beauty captures the mind of the beholder. Journal of Personality and Social Psychology 85:1107-1120.

Maner, Jon K., Matthew T. Gailliot, Aaron D. Rouby, und Saul L. Miller. 2007. Can't take my eyes off you: Attentional adhesion to mates and rivals. Journal of Personality and Social Psycho$\log y$ 93:389-401.

Maurer, Marcus, und Carsten Reinemann. 2006. Medieninhalte. Eine Einführung. Wiesbaden: VS-Verlag.

Mocan, Naci, und Erdal Tekin. 2010. Ugly criminals. Review of Economics and Statistics 92:15-30.

Noelle-Neumann, Elisabeth, Hans Mathias Kepplinger, und Wolfgang Donsbach. 1999. Kampa. Meinungsklima und Medienwirkung im Bundestagswahlkampf 1998. Freiburg: Alber.

Rennenkampff, Anke von. 2004. You look so feminine! When did you fail the last time? Social interaction following the think manager - Think male stereotype. Brandeis Graduate Journal 2:1-7. 
Riniolo, Todd C., Katherine C. Johnson, Tracy R. Sherman, und Julie A. Misso. 2006. Hot or not: Do professors perceived as physically attractive receive higher student evaluations? Journal of General Psychology 133:19-35.

Rosar, Ulrich, und Markus Klein. 2009. Mein(schöner)Prof.de. Die physische Attraktivität des akademischen Lehrpersonals und ihr Einfluss auf die Ergebnisse studentischer Lehrevaluationen. Kölner Zeitschrift für Soziologie und Sozialpsychologie 61:621-645.

Rosar, Ulrich, Markus Klein, und Tilo Beckers. 2008. The frog pond beauty contest. Physical attractiveness and electoral success of the constituency candidates at the North Rhine-Westphalia state election of 2005. European Journal of Political Research 47:64-79.

Schmitt-Beck, Rüdiger. 2000. Politische Kommunikation und Wählerverhalten. Wiesbaden: Westdeutscher Verlag.

Schoen, Harald, und Cornelia Weins. 2005. Der sozialpsychologische Ansatz zur Erklärung von Wahlverhalten. In Handbuch Wahlforschung, Hrsg. Jürgen W. Falter und Harald Schoen, 187 242. Wiesbaden: VS Verlag für Sozialwissenschaften.

Schulz, Winfried. 2007. Politische Kommunikation. Theoretische Ansätze und Ergebnisse empirischer Forschung. Wiesbaden: VS Verlag für Sozialwissenschaften.

Schulz, Winfried, Raimar Zeh, und Oliver Quiring. 2000. Wählerverhalten in der Mediendemokratie. In 50 Jahre Empirische Wahlforschung in Deutschland, Hrsg. Markus Klein, Wolfgang Jagodzinski, Ekkehard Mochmann, und Dieter Ohr, 413-443. Wiesbaden: Westdeutscher Verlag.

Sigelman, Carol K., Dan B. Thomas, Lee Sigelman, und Frederick D. Robich. 1986. Gender, physical attractiveness, and electability: An experimental investigation of voter biases. Journal of Applied Social Psychology 16:229-248.

Sigelman, Lee, Carol K. Sigelman, und Christopher Fowler. 1987. A Bird of a different feather? An experimental investigation of physical attractiveness and the electability of female candidates. Social Psychology Quarterly 50:32-43.

Staab, Joachim-Friedrich. 1986. Direktkandidaten in den Bundestagswahlkämpfen 1969-1983. Erfahrungen im Umgang mit der lokalen und regionalen Presse. Publizistik 31:296-314.

Todorov, Alexander, Anesu N. Mandisodza, Amir Gorem, und Crystal C. Hall. 2005. Inferences of competence from faces predict election outcome. Science 308:1623-1626.

Wilke, Jürgen. 2004. Die Visualisierung der Wahlkampfberichterstattung in Tageszeitungen 19492002. In Visuelle Wahlkampfkommunikation, Hrsg. Thomas Knieper und Marion G. Müller, 210-230. Köln: Halem.

Marcus Maurer, 1969, Dr. phil., zurzeit Gastprofessor für Kommunikationswissenschaft an der Universität Zürich. Forschungsgebiete: Politische Kommunikation, Medienwirkungsforschung, Methoden der empirischen Sozialforschung. Veröffentlichungen: Abschied vom rationalen Wähler. Warum Wahlen im Fernsehen entschieden werden, Freiburg/München 2005 (mit H. M. Kepplinger); Schröder gegen Merkel. Wahrnehmung und Wirkung des TV-Duells 2005 im Ost-West-Vergleich, Wiesbaden 2007 (mit C. Reinemann, J. Maier und M. Maier); Sagen Bilder mehr als tausend Worte? Die Relevanz verbaler und visueller Informationen für die Urteilsbildung über Personen im Fernsehen, Medien \& Kommunikationswissenschaft 57, 2009.

Harald Schoen, 1972, Dr. phil., Professor für Politikwissenschaft an der Universität Bamberg. Forschungsgebiete: Politische Soziologie und Methoden der empirischen Sozialforschung. Veröffentlichungen: Sozialforschung im Internet: Methodologie und Praxis der Online-Befragung, Wiesbaden, 2009 (Hrsg. zusammen mit Nikolaus Jackob und Thomas Zerback); Die Deutschen und die Türkeifrage. Eine Analyse der Einstellungen zum Antrag der Türkei auf Mitgliedschaft in der Europäischen Union, Politische Vierteljahresschrift 49, 2008; Campaigns, Candidate Evaluations, and Vote Choice: Evidence from German Federal Election Campaigns, 1980-2002, Electoral Studies 26, 2007. 